\title{
Importance of Basic Microbiology Research
}

\section{Sujatha $\mathbf{M}^{*}$}

Department of Molecular Biology and Microbiology, Tufts University School of Medicine, USA

*Corresponding author: Sujatha Muralidharan, Department of Molecular Biology and Microbiology, Tufts University School of Medicine, Boston, MA02111, USA, Tel:

\section{Editorial}

Volume 1 Issue 2

Received Date: October 15, 2016

Published Date: October 24, 2016

DOI: $10.23880 /$ oajmb-16000109

(713) 367-8957; E-mail: suju85@hotmail.com

\section{Editorial}

In an era of translational science, proper communication of proposed research significance with respect to human health is one of the prerequisites of successful academic scientific research. Conveying the translational implications of research is essential for scientific publications and for obtaining financial support, both of which are necessary for research progress. Although the reasons for current emphasis on translational "applied science" research is understandable from a political and public standpoint in light of slow progress in developing cures for diseases like cancer and AIDS but it should certainly not be at the expense of "basic science" research.

About 70 years ago, academic institutions including universities were considered the center of basic science research while industry was charged with applying this research towards translational objectives. This is illustrated in the report of Dr. Vannevar Bush to the President in 1945 wherein he stated "The responsibility for basic research in medicine and the underlying sciences, so essential to progress in the war against disease, falls primarily upon the medical schools and universities. These institutions provide the environment which is most conducive to the creation of new scientific knowledge and least under pressure for immediate, tangible results. With some notable exceptions, most research in industry and Government involves application of existing scientific knowledge to practical problems. It is only the colleges, universities, and a few research institutes that devote most of their research efforts to expanding the frontiers of knowledge." [1]. However since the 1980s, when universities were permitted to patent scientific knowledge acquired by federal funding by virtue of the Bay h-Doyle Act, the academic field began to shift towards more translational research which offered universities advantages in terms of public visibility and funding. This is also evidenced in even current academic applications for federal funding whose availability is determined by the extent of translational benefits of proposed research rather than basic scientific discovery and knowledge goals. It should be noted that focusing solely on the practical or therapeutic benefits of a scientific research project undervalues the importance of basic science research and is detrimental for technological and scientific progress at large.

In case of microbiology, while the studies of lifethreatening, pathogenic microbes would be considered significant translational research, it is harder to inspire similar levels of interest in basic science research of other ordinary bacteria that do not cause disease or directly affect human health. It can be a challenge to communicate the importance of such research in an era dominated by translational science. But it should be recognized that many of the tools used by researchers today would not exist if not for basic science research done on ordinary microbes. In fact, the tremendous expansion of the biotech and pharma industry over the years was capacitated by tools developed from basic microbiology research conducted solely for the purpose of scientific discovery and knowledge rather than the development of translational applications. 


\section{Open Access Journal of Microbiology \& Biotechnology}

A very good instance of basic microbiology research leading to development of technology used widely in research today is the restriction endonucleases used for cloning. The phenomenon of restriction enzyme digestion of DNA was first observed by Luria and Human [2] and Bertani and Weigle [3] in 1952-1953 by studying bacteriophages in Escherichia coli and Shigella dysenteriae. In the 1960s, Arber [4] illustrated how bacteria cleverly use "restriction enzymes" to cleave DNA inserted by invading bacteriophages. This seminal discovery was further validated and expanded by Smith [5] wherein he purified and described a restriction endonuclease $\mathrm{R}$ from Haemophilus influenzae that recognized specific T7 DNA sequences and cleaved at specific DNA sites. Nathans and Danna [6] took this a step further and demonstrated the cleavage of SV40 viral genome with this endonuclease into 11 different sized DNA fragments by polyacryamide gel electrophoresis. These enzymes became important tools for determining physical maps of genomes. For these groundbreaking discoveries, Arber, Smith and Nathans jointly received the Nobel Prize in Physiology or Medicine in 1978. Another example of bacterial research contributions to the world of biotechnology tools was the development of polymerase chain reaction (PCR) technique by Mullis [7], for which he received the Nobel prize in 1993, utilizing the heat stable polymerase from thermophile bacteria Thermus aquaticus discovered by Brock and Freeze [8] in the hot springs of Yellowstone National Park in the late 1960s. Restriction endonucleases and PCR techniques continue to be some of the most widely used genetic tools for manipulation and creation of new DNA sequences and prove to be invaluable tools for researchers worldwide even today.

In more recent times, the CRISPR-Cas system has emerged as a powerful tool for genome manipulation. CRISPR (Clustered regularly interspaced short palindromic repeats) was initially reported in Escherichia coli and Mycobacterium tuberculosis [9,10], and further characterized by Mojica as frequent repetitive DNA sequences in the genome of Haloferax mediterranei [11]. Identification of these CRISPR elements in numerous prokaryote genomes by Jansen [12] and Mojica [13] along with the CRISPR-associated Cas genes was a significant breakthrough in identifying the biological relevance of the CRISPR-Cas system [14]. The CRISPR-Cas system is now recognized as a prokaryotic "adaptive" immune defense mechanism that remembers previous infections by integrating short DNA sequences derived from the invading genome into the CRISPR array in the genome. Small guide CRISPR RNAs transcribed from the CRISPR array can then target these same sequences upon recurring infection for cleavage by Cas proteins. The translational applications for the CRISPR-Cas system are numerous; from anti-microbial targeting of pathogenic bacteria to genomic engineering for treatment of genetic diseases.

It is important to realize that these discoveries were made by scientists conducting research on microbes purely for the sake of scientific knowledge rather than for improvement of the human condition. Such open-ended exploration research demonstrates scientific curiosity, one of the hallmarks of a good scientist, and these discoveries illustrate that the cultivation of a healthy scientific curiosity can lead to research resulting in innovative and incredible breakthroughs. It is therefore the duty of the scientific community to encourage junior researchers to engage in curiosity-driven basic science research without the expectation of immediate practical applications and with the goal of contributing to existing scientific knowledge rather than solely for translational applications.

The aforementioned examples of development of scientific technologies illustrate that in a period where translational research is considered more valuable, it is imperative not to disparage basic microbiology research. Basic science research discoveries have enriched our understanding of numerous disease-relevant biomolecules that provide the foundation for subsequent efforts for translational therapeutic design. CRISPR-Cas9, which is quickly becoming a popular tool of research including those of a translational nature, was discovered in basic research studies into environmental adaptation of Haloferax mediterranei to changes in salinity [15]. There are numerous such examples to remind us that while translational "applied science" research has its own importance, it would be to our peril to neglect "basic science" research into prokaryotic cells that have adapted to living on earth for nearly 4 billion years which still contain a wealth of information that should be valued by us in the scientific community.

\section{References}

1. United States (1945) Office of scientific research and development, and Bush V. Science, the endless frontier. A report to the President. US Govt print off Washington,

2. Luria SE, Human ML (1952) A nonhereditary, hostinduced variation of bacterial viruses. Journal bacteriology 64(4): 557-569. 


\section{Open Access Journal of Microbiology \& Biotechnology}

3. Bertani G, Weigle JJ (1953) Host controlled variation in bacterial viruses. J Bacteriol 65(2): 113-121.

4. Arber W (1965) Host-controlled modification of bacteriophage. Annual review of microbiology 19: 365-378.

5. Smith HO, Wilcox KW (1970) A restriction enzyme from Hemophilus influenzae. I. Purification and general properties. Journal of molecular biology 51(2): 379-391.

6. Danna K, Nathans D (1971) Specific cleavage of simian virus 40 DNA by restriction endonuclease of Hemophilus influenzae. Proc Natl Acad Sci USA 68(12): 2913-2917.

7. Mullis KB (1990) Target amplification for DNA analysis by the polymerase chain reaction. Annales de Biologie Clinique 48(8): 579-582.

8. Brock TD, Freeze H (1969) Thermus aquaticus gen. $n$. and sp. n., a nonsporulating extreme thermophile. J Bacteriol 98(1): 289-297.

9. Ishino $\mathrm{Y}$, Shinagawa $\mathrm{H}$, Makino $\mathrm{K}$, Amemura M, Nakata A (1987) Nucleotide sequence of the iap gene, responsible for alkaline phosphatase isozyme conversion in Escherichia coli, and identification of the gene product. J Bacteriol 169(12): 5429-5433.

10. Groenen PM, Bunschoten AE, van Soolingen D, van Embden JD (1993) Nature of DNA polymorphism in the direct repeat cluster of Mycobacterium tuberculosis; application for strain differentiation by a novel typing method. Mol Microbiol 10(5): 10571065.

11. Mojica FJ, Ferrer C, Juez G, Rodriguez VF (1995) Long stretches of short tandem repeats are present in the largest replicons of the Archaea Haloferax mediterranei and Haloferax volcanii and could be involved in replicon partitioning. Molecular microbiology 17(1): 85-93.

12. Jansen R, van Embden JD, Gaastra W, Schouls LM (2002) Identification of a novel family of sequence repeats among prokaryotes. OMICS 6(1): 23-33.

13. Mojica FJ, Diez VC, Soria E, Juez G (2000) Biological significance of a family of regularly spaced repeats in the genomes of Archaea, Bacteria and mitochondria. Mol Microbiol 36(1): 244-246.

14. Jansen R, Embden JD, Gaastra, Schouls LM (2002) Identification of genes that are associated with DNA repeats in prokaryotes. Mol Microbiol 43(6): 15651575.

15. Mojica FJ, JuezG, Rodriguez VF (1993) Transcription at different salinities of Haloferax mediterranei sequences adjacent to partially modified PstI sites. Mol Microbiol 9(3): 613-621. 